\title{
Probabilistic Evaluation of the Integer Least-Squares and Integer Aperture Estimators
}

\author{
S. Verhagen, P.J.G. Teunissen \\ Delft Institute of Earth Observation and Space Systems (DEOS), Delft University of Technology, \\ Kluyverweg 1, 2629 HS Delft, The Netherlands, e-mail: A.A. Verhagen@TUDelft.nl
}

\begin{abstract}
The carrier phase observations start to act as very precise pseudorange observations once the ambiguities are resolved as integers. However, the integer ambiguity estimates should only be used if the reliability of the integer solution is high. The question is then how to assess this reliability. A wellknown a-priori reliability measure is the ambiguity success rate. But even with a high success rate, integer ambiguity validation remains indispensable in order to check whether or not a specific integer solution is sufficiently more likely than any other integer candidate. A solution to the integer validation problem is the use of integer aperture estimation. With this approach an aperture space is defined such that only float samples that fall into this space are fixed to the corresponding integer estimates, otherwise the float solution is maintained. The aperture space is built up of translationally invariant aperture pull-in regions centered at all integers. The size of these pullin regions is determined by the condition of a fixed failure rate.

In this contribution, we will present the probabilistic measures that can be used to assess the reliability of the integer least-squares and the integer aperture ambiguity estimators, as well as the reliability of the corresponding baseline estimators. These probabilities will also be evaluated in the presence of a bias in order to study the bias-robustness of the integer ambiguity estimators. A case study is carried out with several GNSS models, which shows that the integer aperture estimator has some favorable probabilistic properties as compared to integer least-squares estimation, both in the unbiased and in the biased case.
\end{abstract}

Keywords. Integer least-squares, integer aperture estimation, bias-robustness

\section{Integer Estimation}

A GNSS model generally contains real-valued and integer-valued parameters. The latter are the double difference (DD) carrier phase ambiguities, $a$. The first group is referred to as the baseline unknowns, $b$.

The 'float' estimators of the unknown parameters and their variance-covariance (vc-) matrix are obtained after a standard least-squares adjustment:

$$
\left(\begin{array}{c}
\hat{a} \\
\hat{b}
\end{array}\right) ; \quad\left(\begin{array}{cc}
Q_{\hat{a}} & Q_{\hat{a} \hat{b}} \\
Q_{\hat{a} \hat{b}} & Q_{\hat{b}}
\end{array}\right)
$$

In the next step, the float ambiguities are fixed to integer values, which is referred to as ambiguity resolution:

$$
\check{a}=S(\hat{a})
$$

where $S: \mathbb{R}^{n} \longmapsto \mathbb{Z}^{n}$ is the mapping from the $n$-dimensional space of real numbers to the $n$-dimensional space of integers. The optimal result in the sense of maximizing the probability of correct integer estimation (success rate) is obtained using integer least-squares (ILS), cf. Teunissen $(1993,1999)$. The ILS ambiguity estimator is given by:

$$
\check{a}=\arg \min \|\hat{a}-\check{a}\|_{Q_{\hat{a}}}^{2}
$$

An efficient implementation of ILS estimation is provided by the LAMBDA method, see e.g. (Teunissen, 1993; Hofmann-Wellenhoff and Lichtenegger, 2001; Strang and Borre, 1997; Teunissen, 1998; Misra and Enge, 2001).

Finally, the float baseline estimator is adjusted by virtue of its correlation with the ambiguities, giving the 'fixed' baseline estimator:

$$
\check{b}=\hat{b}-Q_{\hat{b} \hat{a}} Q_{\hat{a}}^{-1}(\hat{a}-\check{a})
$$

Ambiguity resolution should only be applied when there is enough confidence in its results. A wellknown reliability measure is the success rate. The success rate equals the probability of correct integer estimation: 


$$
P_{S}=P(\check{a}=a)=P\left(\hat{a} \in S_{a}\right)
$$

with $a$ the true, unknown integer vector. $S_{z}$ is the pull-in region centered at the integer $z$; it contains all real-valued vectors that will be mapped to the same integer $z$. The pull-in region of any admissible integer estimator should fulfill the following conditions:

$$
\begin{cases}\bigcup_{z \in Z^{n}} S_{z}=\mathbb{R}^{n} & \\ \operatorname{Int}\left(S_{u}\right) \bigcap \operatorname{Int}\left(S_{z}\right)=\emptyset, & \forall u, z \in \mathbb{Z}^{n}, u \neq z \\ S_{z}=z+S_{0}, & \forall z \in \mathbb{Z}^{n}\end{cases}
$$

where 'Int' denotes the interior of the subset.

It is generally required that the success rate of integer estimation should be very close to one, or equivalently the failure rate, $P_{f}=1-P_{s}$, should be close to 0 . However, there is no general rule which states how large the success rate should be in order to guarantee a reliable fixed solution. What a user essentially would like to know is the probability that the fixed baseline solution, $\breve{b}$, is better than the float solution, $\hat{b}$, and the effect on the baseline estimate if the ambiguities are fixed incorrectly. Hence, evaluation of the probability that the fixed estimator is closer to the true but unknown $b$ than the float estimator,

$$
P(\|\breve{b}-b\| \leq\|\hat{b}-b\|),
$$

is not enough, since even if this probability is larger than 0.5 it does not tell how large the position error can become in the cases that $b$ is not better than $\hat{b}$. Therefore, also the probabilities that the position error is larger than a certain value, $\beta$, should be considered:

$$
P(|| \check{b}-b \| \geq \beta) \quad \text { and } \quad P(\|\hat{b}-b\| \geq \beta)
$$

Unfortunately, none of the probabilities in equation (7) and (8) can be evaluated exactly. In Verhagen (2005) it was empirically shown that it can be expected that $P(\|\breve{b}-b\| \geq \beta)>P(\|\hat{b}-b\| \geq \beta)$ for small $\beta$, but that for large $\beta$ the opposite is true. This is an indication that the position error due to incorrect ambiguity fixing may be very large. This can be further evaluated by considering the probabilities that the position errors are larger than $\beta$ if the ambiguities are known to be fixed incorrectly. For instance, if the failure rate $P_{f}$ is considered large and $P(\|\breve{b}-b\| \geq$ $\left.\beta \mid \hat{a} \notin S_{a}\right)>P\left(|| \hat{b}-b|| \geq \beta \mid \hat{a} \notin S_{a}\right)$ for all values of $\beta$, then the float solution should be preferred.

\section{Integer Aperture Estimation}

In practice, a user may decide not to use the fixed solution if the failure rate is too high. This gives rise to the thought that it might be interesting to use an ambiguity estimator defined such that three situations are distinguished: success if the ambiguity is fixed correctly, failure if the ambiguity is fixed incorrectly, and undecided if the float solution is maintained. This can be accomplished by dropping the condition that there are no gaps between the pull-in regions, so that the only conditions on the pull-in regions are that they should be disjunct and translationally invariant. Then integer estimators can be determined that somehow regulate the probability of each of the three situations mentioned above.

The new class of ambiguity estimators was introduced in Teunissen (2003), and is called the class of Integer Aperture (IA) estimators. The IA ambiguity estimator is given by:

$$
\bar{a}=\left\{\begin{array}{cc}
\check{a} & \text { if } \hat{a} \in \Omega \\
\hat{a} & \text { otherwise }
\end{array}\right.
$$

with

$\begin{cases}\bigcup_{z \in \mathbb{Z}^{n}} \Omega_{z}=\Omega & \\ \operatorname{Int}\left(\Omega_{u}\right) \cap \operatorname{Int}\left(\Omega_{z}\right)=\emptyset, & \forall u, z \in \mathbb{Z}^{n}, u \neq z \\ \Omega_{z}=z+\Omega_{0}, & \forall z \in \mathbb{Z}^{n}\end{cases}$

$\Omega \subset \mathbb{R}^{n}$ is called the aperture space. It follows that this space is built up of the $\Omega_{z}$, which will be referred to as aperture pull-in regions.

Besides the success and failure rates, in this case also the undecided rate, $P_{u}$, must be considered:

$$
\begin{aligned}
P_{s} & =P\left(\hat{a} \in \Omega_{a}\right) \\
P_{f} & =P\left(\hat{a} \in \Omega \backslash\left\{\Omega_{a}\right\}\right) \\
P_{u} & =P\left(\hat{a} \notin \Omega_{a}\right)=1-P_{s}-P_{f}
\end{aligned}
$$

The approach of integer aperture estimation allows us to choose a threshold for the failure rate, and then determine the size of the aperture pull-in regions such that indeed the failure rate will be equal to or below this threshold. So, applying this approach means that implicitly the ambiguity estimate is validated using a sound criterion. However, there are still several options left with respect to the choice of the shape of the aperture pull-in regions. In the class of integer estimators the ILS estimator is optimal as it maximizes the success rate. Likewise, the Optimal IA estimator can be defined as the one which maximizes the 
success rate on the condition that the failure rate is equal to a fixed value $\alpha$, cf. Teunissen (2005). Hence, the aperture pull-in region, $\Omega_{0}$ is obtained by solving:

$$
\max _{\Omega_{0}} P_{s} \quad \text { subject to } P_{f}=\alpha
$$

Note that for $\alpha \geq P\left(\hat{a} \notin S_{a}\right)$, OIA estimation is identical to ILS estimation.

The unconditional success rate, $P(\bar{a}=a)$, is not very useful as a reliability measure for integer aperture estimation, since there is always a probability of undecidedness. Hence, the unconditional success rate will always be lower than or, in very favorable conditions, equal to the success rate of ILS estimation, cf. equation (5). But at the same time the failure rate is always smaller or equal. Instead of the unconditional success rate one should therefore consider the conditional success rate, which is the probability that an integer outcome is correct:

$$
P_{s \mid \hat{a} \in \Omega}=P(\bar{a}=a \mid \hat{a} \in \Omega)=\frac{P_{S}}{P_{s}+P_{f}}
$$

It can be easily shown that with Optimal IA estimation also the conditional success rate is maximized.

Obviously, $P_{s}+P_{f}$ equals the probability of an integer outcome. Hence, the conditional success rate will be close to one when the failure rate is chosen close to zero and $P_{s}>>P_{f}$. This implies that one can have a very high confidence indeed in the correctness of the integer outcomes of the OIA estimator, even for modest values of the unconditional success rate. With ILS estimation such a high confidence can only be reached once the ILS success rate is close to one, cf. Section 1.

In order to compare the reliability of the integer aperture baseline estimator, $\bar{b}$, with that of the float and fixed estimators, probabilities equivalent to those in equations (7) and (8) can be evaluated:

$$
P(\|\bar{b}-b\| \leq\|\hat{b}-b\|) \text { and } P(\|\bar{b}-b\| \geq \beta)
$$

\section{Effect of a Bias}

So far, the reliability of integer estimation has only been considered under the assumption that no model error is present. However, incorrect ambiguity fixing can also be due to the presence of a bias. Assume that the observations are biased, and the corresponding biased float solution is given as:

$$
\left(\begin{array}{c}
\hat{b}_{\nabla} \\
\hat{a}_{\nabla}
\end{array}\right)=\left(\begin{array}{c}
\hat{b}+\nabla \hat{b} \\
\hat{a}+\nabla \hat{a}
\end{array}\right)
$$

The corresponding biased fixed solution will be denoted as $\check{a}_{\nabla}$ and $\breve{b}_{\nabla}$. Note that:

$$
\check{a}_{\nabla}=\check{a}+\nabla \check{a}=S\left(\hat{a}_{\nabla}\right) \neq S(\hat{a})+S(\nabla \hat{a})
$$

Hence, the effect of the bias on the fixed ambiguities, $\nabla \check{a}$ does not only depend on the bias itself, but also on the float solution. The bias in the fixed baseline solution follows as:

$$
\nabla \check{b}=\check{b}_{\nabla}-\check{b}=\nabla \hat{b}-Q_{\hat{b} \hat{a}} Q_{\hat{a}}^{-1}(\nabla \hat{a}-\nabla \check{a})
$$

Of course it is not possible to make general statements on how a bias in the float solution will affect the fixed baseline solution, since that depends on the type and the size of the bias as well as on the normal uncertainty of the float solution. The first question is how the float solution itself is affected by the bias: are the ambiguities and baseline both severely affected, or not? The next question is how the bias in the float ambiguities affects the probability of correct fixing. The probability of correct ambiguity fixing in the presence of a bias is called the bias-affected success rate, cf. Teunissen (2001), and can be computed once the bias in the float ambiguities, $\nabla \hat{a}$, is known:

$$
P_{s}^{\nabla}=P\left(\check{a}_{\nabla}=a\right)=P\left(\hat{a}+\nabla \hat{a} \in S_{a}\right)
$$

If the bias-affected success rate is close to the success rate, obviously the ambiguity estimator is not sensitive to the bias. However, this does not tell us how the fixed baseline solution is affected. As can be seen in equation (17), the bias in the fixed baseline solution depends on $\nabla \hat{b}, \nabla \hat{a}$ and $\nabla \check{a}$. It is interesting though, to evaluate the probabilities in equations (7) and (8) for the biased float and fixed estimators, and to compare these probabilities with those of the unbiased equivalents.

Finally, it is interesting to study the bias sensitivity of the OIA estimator. The bias-affected success rates, both the conditional and the unconditional, will be lower than in the unbiased situation. This becomes clear from the two-dimensional example in Figure 1, where the contours of the PDF of the float ambiguities are shown for the unbiased situation on the left, and for two biased cases in the center and on the right. The smaller regions inside the ILS pull-in regions (hexagons) depict the aperture pull-in regions corresponding to a failure rate of 0.005 (in the unbiased case).

It can be expected that if the precision of the float solution is not so good, and consequently the integer least-squares success rate is low, that relatively 

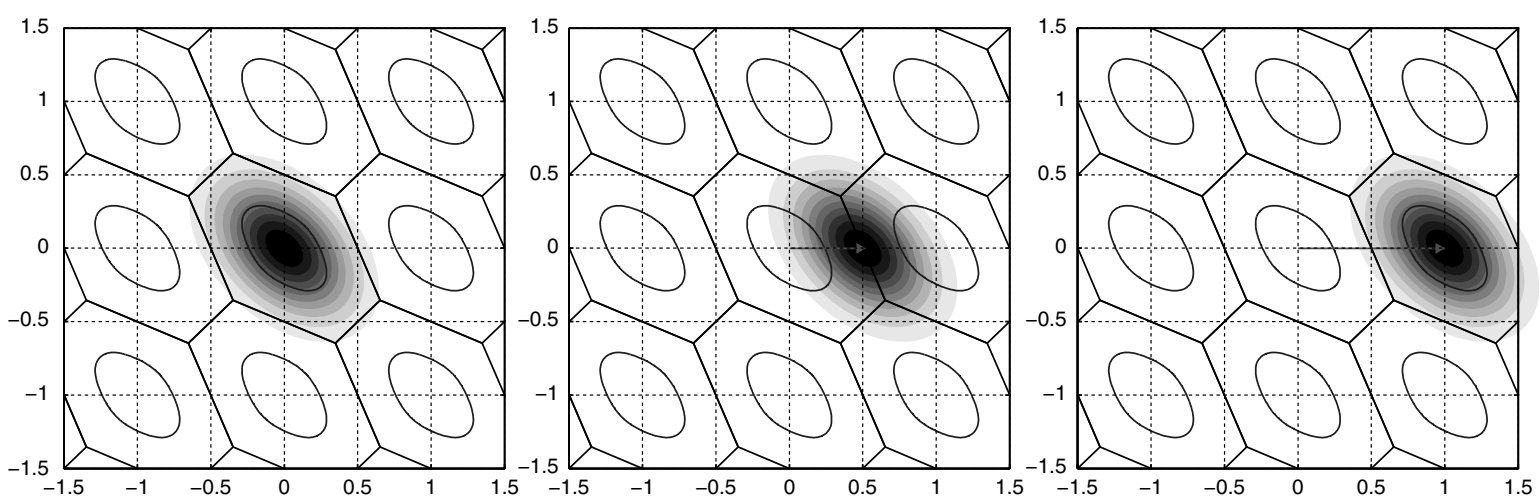

Fig. 1. Probability distributions of the biased float ambiguities. The true integer is $a=0$, the bias is depicted with the arrow. The aperture pull-in regions and ILS pull-in regions (hexagons) are shown. Left: no bias; Center: bias of [0.5 0]; Right: bias of [1 0].

small biases in the float ambiguities will be absorbed in the noise. In that case there is namely a high probability of large ambiguity residuals, and thus a large undecided rate with IA estimation. This remains unchanged in the presence of the bias.

The opposite may be true for stronger models. In that case it really depends on the size of the bias whether or not the IA estimator is sensitive to it. If the bias is such that almost all probability mass of the float ambiguities is transferred to the wrong pullin region, as in the right panel of Figure 1, this will certainly not lead to more rejected fixed ambiguities. In fact, there is a very high probability then that the ambiguities are fixed incorrectly. If the bias causes a high probability that the float ambiguity will be close to the boundary of a pull-in region, as in the middle panel of Figure 1, there is some sensitivity to the bias, as more samples will be rejected. At the same time, the bias-affected success rate will be lower and the bias-affected failure rate will be higher. If the bias is such that most of the probability mass is still in the correct aperture pull-in region $\Omega_{a}$ there is a low sensitivity to the bias, but that is good, since then the bias-affected success rate will be close to the success rate in the unbiased situation.

Apart from the conditional and unconditional success rates, also the probabilities in Eq.(14) should be evaluated for the biased IA estimator.

\section{Case Study}

The probabilities presented in this paper are evaluated for a dual-frequency GPS model, with four satellites visible. The results are representative for practical situations; other models have been considered but the results are not shown here. For the biased situation, an unmodeled ionospheric delay is considered; the resulting bias in the float baseline solution is $\|\nabla \hat{b}\|=4 \mathrm{~cm}$.

The results are shown in Table 1 and Figure 2. Some important conclusions will be discussed here.

1. If $P_{s}>>P_{f}$ the fixed baseline estimator is better than the float baseline estimator, but with the fixed estimator there is a higher probability of a very large position offset than with the float estimator due to its multi-modal distribution.

From the probabilities in Table 1 follows that in most cases $\|\breve{b}-b\| \leq\|\hat{b}-b\|$. Also from the graphs in Figure 2 it follows that there is indeed a high probability of $\breve{b}$ being better than $\hat{b}$, but there is also a higher probability that $\breve{b}$ is very far off the true $b$. It follows that if $\hat{a} \notin S_{a}$ there is a risk of a very large position offset $\|\breve{b}-b\|$ as compared to $\|\hat{b}-b\|$.

2. Ambiguities should be fixed if one can have enough confidence in their correctness.

In all cases that the ambiguities are fixed correctly $\|\breve{b}-b\|$ will be very small, as follows from the

Table 1. ILS and OIA probabilities, with $P_{b}=P(\|\bar{b}-b\|$ $\leq\|\hat{b}-b\| \mid \hat{a} \in \Omega)$ and $P_{b}^{\nabla}=(\|\bar{b} \nabla-b\| \leq\|\hat{b} \nabla-b\|$ $\mid \hat{a} \nabla \in \Omega$ ). Note that for ILS $\Omega=\mathbb{R}^{n}$

\begin{tabular}{llll}
\hline & ILS & OIA & \\
\hline$P_{f}$ & 0.183 & 0.050 & 0.005 \\
$P_{S}$ & 0.817 & 0.655 & 0.330 \\
$P_{s \mid \hat{a} \in \Omega}$ & 0.817 & 0.929 & 0.985 \\
$P_{b}$ & 0.862 & 0.946 & 0.989 \\
$P_{f}^{\nabla}$ & 0.689 & 0.275 & 0.056 \\
$P_{s}^{\nabla}$ & 0.311 & 0.169 & 0.046 \\
$P_{s \mid \hat{a} \in \Omega}^{\nabla}$ & 0.311 & 0.381 & 0.450 \\
$P_{b}^{\nabla}$ & 0.549 & 0.604 & 0.653 \\
\hline
\end{tabular}



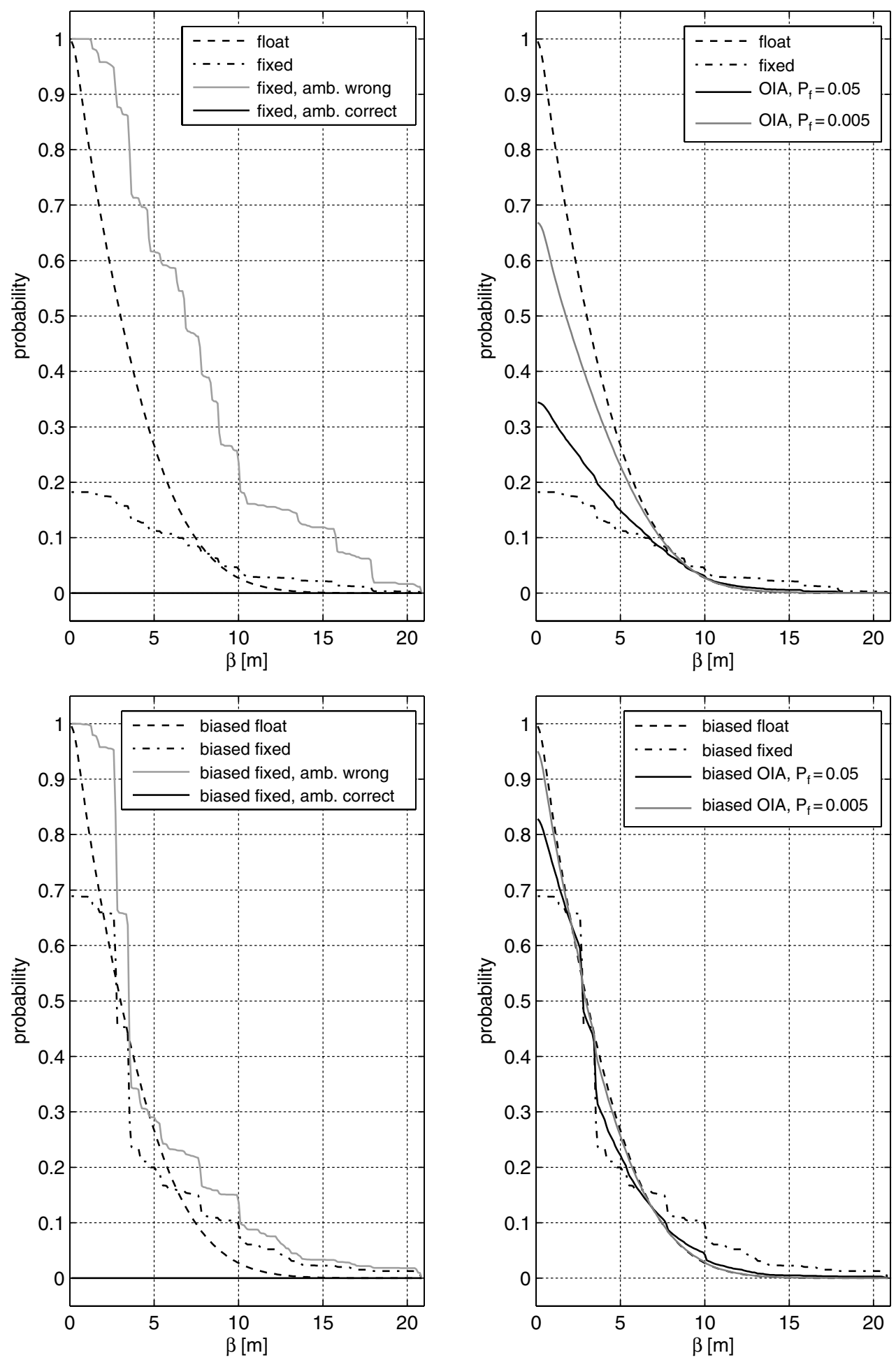

Fig. 2. Baseline probabilities $P\left(\left\|b_{e}-b\right\| \geq \beta\right)$, where $b_{e}$ is either the float, fixed or OIA baseline estimator. The probabilities conditioned on the ambiguities being fixed correctly and incorrectly are also shown for the float and fixed estimators in the left panels. Top: unbiased situation. Bottom: biased situation. 
graph of the probability $P\left(\|\check{b}-b\| \geq \beta \mid \hat{a} \in S_{a}\right)$ in Figure 2 (top left).

This conclusion confirms the importance of integer ambiguity validation and the need for appropriate reliability measures for the fixed estimator, since a user does not want to take the risk of using a fixed position which is much further away from the true position than the float estimate.

IA estimation provides the means for using the fixed estimates only if there is enough confidence in their correctness. The graphs in Figure 2 show that indeed the risk of a very large position offset due to incorrect fixing is smaller than with the fixed estimator. At the same time there is a lower probability of $\|\bar{b}-b\|$ being small than of $\|\breve{b}-b\|$ being small. The IA estimator can be considered as a good compromise between the float and fixed estimator. Furthermore it follow that $\bar{b}$ is generally closer to the true $b$ than $\hat{b}$ if $\hat{a} \in \Omega$, and that

$$
P(\|\bar{b}-b\| \leq\|\hat{b}-b\| \mid \hat{a} \in \Omega)
$$

increases with a decreasing failure rate. Hence, the probability in equation (19) will be lower than with ILS estimation if the ILS failure rate is larger than the user-defined value $\alpha$. Apparently, one can be quite sure that if the fixed solution is accepted it is indeed better than the float solution. Finally note that the conditional success rates are higher than the integer least-squares success rate.

3. If the float solution is biased, the probability of the fixed baseline estimator being better than the float estimator becomes lower than in the unbiased situation.

The baseline probabilities are all lower in the biased situation, except for the probability $P(\| \breve{b}-$ $b\|\leq\| \hat{b}-b \| \mid \hat{a} \notin S_{a}$ ), which means that there is a somewhat higher probability that the fixed baseline estimator is better than the float if the ambiguities are fixed incorrectly.

4. Integer Aperture estimation does provide some protection, but is not a safeguard against biases.

The bias in the float baseline solution is small $(4 \mathrm{~cm})$ and not visible in the corresponding graph in Figure 2 (bottom left). However, there is a much higher probability of a large offset $\left\|\breve{b}_{\nabla}-b\right\|$ as compared to the unbiased situation. If, on the other hand, the ambiguities are fixed correctly, the fixed baseline estimator still performs much better than its float counterpart.
Hence, also in the biased situation IA estimation may offer a good compromise between the float and fixed estimator, since the undecided rate will be higher. As expected the probability that $\left\|\bar{b}_{\nabla}-b\right\|$ $\leq\left\|\hat{b}_{\nabla}-b\right\|$ if $\bar{a}=\check{a}$ is smaller than in the unbiased situation, but still is higher than the probability that $\left\|\breve{b}_{\nabla}-b\right\| \leq\left\|\hat{b}_{\nabla}-b\right\|$.

Note that in the biased situation the failure rate is larger than the threshold value due to the incorrect model assumption. But, of course, the bias-affected failure rate is still lower than the corresponding biasaffected ILS failure rate.

In Figure 2 it can be seen that also in the biased situation, the probabilities $P\left(\left\|\bar{b}_{\nabla}-b\right\| \geq \beta\right)$ are in between those of the float and fixed estimators. The probability is almost equal to that of the float estimator when the failure rate is chosen small, since then the bias-affected success rate is small too, and thus the undecided rate is large. Obviously, the biased fixed estimator has a higher probability of a large position offset as compared to the biased IA estimator. The difference is even larger than in the unbiased situation.

\section{References}

Hofmann-Wellenhoff, B. and Lichtenegger, H. (2001). Global Positioning System: Theory and Practice. Springer-Verlag, Berlin, 5 edition.

Misra, P. and Enge, P. (2001). Global Positioning System: Signals, Measurements, and Performance. Ganga-Jamuna Press, Lincoln, MA.

Strang, G. and Borre, K. (1997). Linear Algebra, Geodesy, and GPS. Wellesley-Cambridge Press, Wellesley MA.

Teunissen, P. J. G. (1993). Least squares estimation of the integer GPS ambiguities. Invited lecture, Section IV Theory and Methodology, IAG General Meeting, Beijing.

Teunissen, P. J. G. (1998). GPS carrier phase ambiguity fixing concepts. In: PJG Teunissen and Kleusberg A, GPS for Geodesy, Springer-Verlag, Berlin.

Teunissen, P. J. G. (1999). An optimality property of the integer least-squares estimator. Journal of Geodesy, 73(11), 587-593.

Teunissen, P. J. G. (2001). Integer estimation in the presence of biases. Journal of Geodesy, 75, 399-407.

Teunissen, P. J. G. (2003). Integer aperture GNSS ambiguity resolution. Artificial Satellites, 38(3), 79-88.

Teunissen, P. J. G. (2005). GNSS ambiguity resolution with optimally controlled failure-rate. Artificial Satellites, 40(4), 219-227.

Verhagen, S. (2005). The GNSS integer ambiguities: estimation and validation. Ph.D. thesis. Publications on Geodesy, 58, Netherlands Geodetic Commission, Delft. 